\title{
Forced Oscillation of Neutral Impulsive Parabolic Partial Differential Equations with Continuous Distributed Deviating Arguments
}

\author{
Guangjie Liu ${ }^{1,2}$, Chengyong Wang1 \\ ${ }^{1}$ School of Mathematics and Computer Science, Hubei University of Arts and Science, Xiangyang, China \\ ${ }^{2}$ School of Mathematics and Physics, China University of Geosciences, Wuhan, China \\ Email: guangjieliu@foxmail.com
}

Received 26 October 2014; revised 27 November 2014; accepted 18 December 2014

Copyright (C) 2014 by authors and OALib.

This work is licensed under the Creative Commons Attribution International License (CC BY).

http://creativecommons.org/licenses/by/4.0/

\section{(c) (i) Open Access}

\section{Abstract}

This paper investigated oscillatory properties of solutions for nonlinear parabolic equations with impulsive effects under two different boundary conditions. By using integral averaging method, variable substitution and functional differential inequalities, we established several sufficient conditions. At last, we provided two examples to illustrate the results.

\section{Keywords}

Forced Oscillation, Parabolic Equation, Impulsive, Neutral Type, Continuous Distributed Deviating Arguments

\section{Subject Areas: Integral Equation, Mathematical Analysis, Partial Differential Equation}

\section{Introduction}

In this article, we discuss forced oscillatory properties of solutions for the nonlinear impulsive parabolic equations of neutral type.

$$
\begin{aligned}
& \frac{\partial}{\partial t}\left[u(t, x)+\int_{\alpha}^{\beta} g(t, \xi) u(\rho(t, \xi), x) \mathrm{d} \eta(\xi)\right] \\
& =a(t) \Delta u(t, x)+\sum_{i=1}^{l} b_{i}(t) \Delta u\left(\tau_{i}(t), x\right)-\int_{\gamma}^{\delta} q(t, x, \zeta) f(u(\sigma(t, \zeta), x)) \mathrm{d} \omega(\zeta) \\
& \quad+F(t, x), \quad t \neq t_{k},(t, x) \in \mathbb{R}^{+} \times \Omega=G,
\end{aligned}
$$


with the boundary conditions

$$
u\left(t_{k}^{+}, x\right)-u\left(t_{k}^{-}, x\right)=b_{k} u\left(t_{k}, x\right), \quad k=1,2, \cdots
$$

$$
\begin{gathered}
u=0,(t, x) \in \mathbb{R}^{+} \times \partial \Omega, \\
\frac{\partial u}{\partial n}+c u=0,(t, x) \in \mathbb{R}^{+} \times \partial \Omega
\end{gathered}
$$

and the initial condition

$$
u(t, x)=\Phi(t, x),(t, x) \in[-\lambda, 0] \times \Omega .
$$

Here $\Omega \subset \mathbb{R}^{N}$ is a bounded domain with boundary $\partial \Omega$ smooth enough and $n$ is the unit exterior normal vector of $\partial \Omega, \max _{t \in \mathbb{R}^{+}}\left\{t-\rho(t, \xi), t-\tau_{i}(t), t-\sigma(t, \zeta)\right\} \leq \lambda$ a positive constant, $\Phi(t, x) \in C^{2}([-\lambda, 0] \times \Omega,) \mathbb{R}$.

We will use the following conditions.

(H1) $a(t), b_{i}(t) \in P C\left(\mathbb{R}^{+}, \mathbb{R}^{+}\right), \tau_{i}(t) \in C\left(\mathbb{R}^{+}, \mathbb{R}\right), g(t, \xi) \in P C\left(\mathbb{R}^{+} \times[\alpha, \beta], \mathbb{R}^{+}\right)$, $\rho(t, \xi) \in C\left(\mathbb{R}^{+} \times[\alpha, \beta], \mathbb{R}\right), \quad \sigma(t, \zeta) \in C\left(\mathbb{R}^{+} \times[\gamma, \delta], \mathbb{R}\right), \quad q(t, x, \zeta) \in C\left(\mathbb{R}^{+} \times \bar{\Omega} \times[\gamma, \delta], \mathbb{R}^{+}\right)$, such that $t-\rho(t, \xi)>0, t>t-\imath>\sigma(t, \zeta), \lim _{t \rightarrow \infty} \min _{\xi \in[\alpha, \beta]} \rho(t, \xi)=\infty, \lim _{t \rightarrow \infty} \tau_{i}(t)=\infty$, $\lim _{t \rightarrow \infty} \min _{\zeta \in[\gamma, \delta]} \sigma(t, \zeta)=\infty$, where $\imath$ is a constant, $P C$ denote the class of functions which are piecewise continuous in $t$ with discontinuities of first kind only at $t=t_{k}$, and left continuous at $t=t_{k}, k=1,2, \cdots$, $\rho\left(t_{k}, \xi\right) \neq t_{j}$ for $j<k, \quad j=1,2, \cdots, g\left(t_{k}^{+}, \xi\right)=\left(1+b_{k}\right) g\left(t_{k}^{-}, \xi\right)$.

(H2) $-\eta(\xi), \omega(\zeta)$ are nondecreasing functions on $[\alpha, \beta]$ and $[\gamma, \delta]$, respectively; $f(u) \in C(\mathbb{R}, \mathbb{R})$, $f(u) / u \geq C,-\int_{\alpha}^{\beta} g(t, \xi) \mathrm{d} \eta(\xi) \leq h_{0}<1$, where $h_{0}$ and $C$ are positive constants, $u \neq 0 ; c>0, \quad b_{k} \geq 0$, $k=1,2, \cdots, \int_{\Omega} \varphi(x) F(t, x) \mathrm{d} x \leq 0, \lim _{k \rightarrow \infty} t_{k}=\infty, 0<t_{1}<t_{2}<\cdots$.

(H3) $u(t, x)$ is piecewise continuous in $t$ with discontinuities of first kind only at $t=t_{k}$, and left continuous at $t=t_{k}, u\left(t_{k}, x\right)=u\left(t_{k}^{-}, x\right), \quad k=1,2, \cdots$.

Let us construct the sequence $\left\{\bar{t}_{k}\right\}=\left\{t_{k}\right\} \cup\left\{t_{j \xi}\right\} \cup\left\{t_{j \tau}\right\} \cup\left\{t_{j \zeta}\right\}$, where $\left\{t_{j \xi}\right\}=\left\{t \mid \rho(t, \xi)=t_{j}\right\}$, $\left\{t_{j \tau}\right\}=\left\{t \mid \tau_{i}(t)=t_{j}\right\}, \quad\left\{t_{j \zeta}\right\}=\left\{t \mid \sigma(t, \zeta)=t_{j}\right\}$ and $\bar{t}_{k}<\bar{t}_{k+1}, \quad i=1,2, \cdots, l ; k, j=1,2, \cdots$.

We introduce the notations:

$$
\begin{gathered}
\Gamma_{k}=\left\{(t, x): t \in\left(\bar{t}_{k}, \bar{t}_{k+1}\right), x \in \Omega\right\}, \quad \Gamma=\bigcup_{k=0}^{\infty} \Gamma_{k}, \\
\bar{\Gamma}_{k}=\left\{(t, x): t \in\left(\bar{t}_{k}, \bar{t}_{k+1}\right), x \in \bar{\Omega}\right\}, \bar{\Gamma}=\bigcup_{k=0}^{\infty} \bar{\Gamma}_{k} \\
v(t)=\int_{\Omega} \varphi(x) u(t, x) \mathrm{d} x, Q(t, \zeta)=\min _{x \in \bar{\Omega}} q(t, x, \zeta) .
\end{gathered}
$$

The solution $u \in C^{2}(\Gamma) \cap C^{1}(\bar{\Gamma})$ of problems (1), (3) ((4)) is called non-oscillatory in the domain $G$ if it is either eventually positive or eventually negative. Otherwise, it is called oscillatory.

As is well-known, oscillatory properties of partial differential equations are very important both in theory and application. The developing theory of partial differential equations has been applied in many fields, such as biology, chemistry, engineering, theoretical physics, generic repression, climate model, and so on. In the last few years, the fundamental theories of partial differential equations with deviating arguments have undergone intensive development. We can see lots of studies [1]-[5] which have been done under the assumption that the state variables and system parameters change continuously. However, one may easily visualize situations in nature where abrupt change such as harvesting or disasters may occur [6]. These phenomena are short-time perturbations whose duration is negligible in comparison with the duration of the whole evolution process. Consequently, it is natural to assume, in modelling these problems that these perturbations act instantaneously, that is, in the form of impulses. 
In 1991, the first paper [7] on this class of equations was published. However, we only find a few of papers on oscillation theory of impulsive partial differential equations. Recently, Bainov, Minchev, Fu, and Liu [8]-[12] investigated the oscillation of solutions of impulsive partial differential equations with or without deviating arguments, and Tanaka, Luo, and Shoukaku [13]-[15] discussed the oscillation of solutions of partial differential equations with continuous distributed deviating arguments. However, there is a scarcity in the study of forced oscillation theory of nonlinear impulsive parabolic equations of neutral type with continuous distributed deviating arguments.

\section{Oscillation Properties of the Problem (1), (4)}

For the main result of this article, we need the following lemma.

Lemma 2.1. Let $b_{0}$ and $\varphi(x)$ be the minimum eigenvalue and the corresponding eigenfunction, respectively, of the problem

$$
\begin{gathered}
\Delta \varphi(x)+b \varphi(x)=0, \quad x \in \Omega, \\
\varphi(x)=0, \text { or } \frac{\partial \varphi}{\partial x}+c \varphi(x)=0, x \in \partial \Omega,
\end{gathered}
$$

where $c$ is a positive constant and $n$ is outer normal vector of $\partial \Omega$ at point $x$. Then $b>0$ and $\varphi(x)>0$ for $x \in \Omega$.

Lemma 2.2. [16] Let $\rho=$ const $>0, a_{0}(t), p(t) \in([0,+\infty), R)$ be locally summable functions and $p(t)>0 ; y\left(t_{k}\right)=y\left(t_{k}^{-}\right), \quad k=1,2, \cdots$. If the following condition is satisfied

$$
\liminf _{t \rightarrow+\infty} \int_{t-\rho}^{t} p(s) \exp \left(\int_{s-\rho}^{s} a_{0}(r) \mathrm{d} r\right) \prod_{s-\rho<t_{k}<s}\left(1+d_{k}\right)^{-1} \mathrm{~d} s>\frac{1}{\mathrm{e}},
$$

then the following differential inequality has no eventually positive solution.

$$
\begin{gathered}
y^{\prime}(t)+a_{0}(t) y(t)+p(t) y(t-\rho) \leq 0, t \geq 0, t \neq t_{k} \\
y\left(t_{k}^{+}\right)-y\left(t_{k}^{-}\right)=d_{k} y\left(t_{k}\right), k=1,2, \cdots .
\end{gathered}
$$

The following theorem is the first main result of this article.

Theorem 2.1. Suppose that the conditions (H1)-(H3) and the following condition (5) hold

$$
\liminf _{t \rightarrow+\infty} \int_{t-l}^{t} \exp \left(\int_{s-l}^{s} b_{0} a(r) \mathrm{d} r\right) \prod_{\sigma(t, \zeta) \leq t_{k}<t}\left(1+b_{k}\right)^{-1} \mathrm{~d} s \int_{\gamma}^{\delta} C\left(1-h_{0}\right) Q(s, \zeta) \mathrm{d} \omega(\zeta)>\frac{1}{\mathrm{e}} .
$$

Then each solution of the problem (1)-(3) oscillates in $G$.

Proof. Suppose that the assertion is not true and $u(t, x)$ is a non-oscillatory solution of problem (1), (3) in $G$. Without loss of generality, we may assume that there exists a $t_{0}>0$ such that $u(t, x)>0, u(\rho(t, \xi), x)>0$, $u\left(\tau_{i}(t), x\right)>0, \quad i=1,2, \cdots, l, u(\sigma(t, \zeta), x)>0$, for any $(t, x) \in\left[t_{0},+\infty\right) \times \Omega$.

For $t \geq t_{0}, t \neq \bar{t}_{k}, \quad k=1,2, \cdots$, multiplying (1) by $\varphi(x)$ and then integrating it with respect to $x$ over $\Omega$ yields

$$
\begin{aligned}
& \frac{\mathrm{d}}{\mathrm{d} t}\left[\int_{\Omega} \varphi(x) u(t, x) \mathrm{d} x+\int_{\alpha}^{\beta} g(t, \xi) \mathrm{d} \eta(\xi) \int_{\Omega} \varphi(x) u(\rho(t, \xi), x) \mathrm{d} x\right] \\
& =a(t) \int_{\Omega} \varphi(x) \Delta u \mathrm{~d} x+\sum_{i=1}^{l} b_{i}(t) \int_{\Omega} \varphi(x) \Delta u\left(\tau_{i}(t), x\right) \mathrm{d} x \\
& \quad-\int_{\gamma}^{\delta} \mathrm{d} \omega(\zeta) \int_{\Omega} \varphi(x) q(t, x, \zeta) f(u(\sigma(t, \zeta), x)) \mathrm{d} x+\int_{\Omega} \varphi(x) F(t, x) \mathrm{d} x .
\end{aligned}
$$

By Green's formula and the boundary condition, we have

It follows that

$$
\int_{\Omega} \varphi(x) \Delta u \mathrm{~d} x-\int_{\Omega} u \Delta \varphi(x) \mathrm{d} x=\int_{\partial \Omega} \frac{\partial \varphi}{\partial n} u \mathrm{~d} s-\int_{\partial \Omega} \frac{\partial u}{\partial n} \varphi \mathrm{d} s=0 .
$$

$$
\int_{\Omega} \varphi(x) \Delta u \mathrm{~d} x=\int_{\Omega} u \Delta \varphi(x) \mathrm{d} x=-b_{0} \int_{\Omega} u \varphi(x) \mathrm{d} x
$$




$$
\int_{\Omega} \varphi(x) \Delta u\left(\tau_{i}(t), x\right) \mathrm{d} x=\int_{\Omega} u\left(\tau_{i}(t), x\right) \Delta \varphi(x) \mathrm{d} x=-b_{0} \int_{\Omega} u\left(\tau_{i}(t), x\right) \varphi(x) \mathrm{d} x .
$$

From condition (H2), we can easily obtain

$$
\begin{gathered}
\int_{\Omega} \varphi(x) q(t, x, \zeta) f(u(\sigma(t, \zeta), x)) \mathrm{d} x \geq C Q(t, \zeta) \int_{\Omega} \varphi(x) u(\sigma(t, \zeta), x) \mathrm{d} x . \\
\int_{\Omega} \varphi(x) F(t, x) \mathrm{d} x \leq 0 .
\end{gathered}
$$

From the above it follows that

$$
\begin{aligned}
& \frac{\mathrm{d}}{\mathrm{d} t}\left[v(t)+\int_{\alpha}^{\beta} g(t, \xi) v(\rho(t, \xi)) \mathrm{d} \eta(\xi)\right]+b_{0} a(t) v(t) \\
& +b_{0} \sum_{i=1}^{l} b_{i}(t) v\left(\tau_{i}(t)\right)+C \int_{\gamma}^{\delta} Q(t, \zeta) v(\sigma(t, \zeta)) \mathrm{d} \omega(\zeta) \leq 0 .
\end{aligned}
$$

In inequality (6), set $w(t)=\prod_{t_{0} \leq t_{k}<t}\left(1+b_{k}\right)^{-1} v(t)$, we can obtain the following results: 1) $w(t)$ is continuous on $\left.\left[t_{0},+\infty\right) ; 2\right)$ Inequality (6) has no eventually positive solution if the following inequality (7) has no eventually positive solution.

where

$$
\begin{aligned}
& \frac{\mathrm{d}}{\mathrm{d} t}\left[w(t)+\int_{\alpha}^{\beta} G(t, \xi) w(\rho(t, \xi)) \mathrm{d} \eta(\xi)\right]+b_{0} a(t) w(t) \\
& +b_{0} \sum_{i=1}^{l} B_{i}(t) w\left(\tau_{i}(t)\right)+C \int_{\gamma}^{\delta} Q_{0}(t, \zeta) w(\sigma(t, \zeta)) \mathrm{d} \omega(\zeta) \leq 0, \quad t \geq t_{0}, t \neq \overline{t_{k}},
\end{aligned}
$$

$$
\begin{aligned}
& G(t, \xi)=\prod_{\rho(t, \xi) \leq t_{k}<t}\left(1+b_{k}\right)^{-1} g(t, \xi), B_{i}(t)=\prod_{\tau_{i}(t) \leq t_{k}<t}\left(1+b_{k}\right)^{-1} b_{i}(t), \\
& Q_{0}(t, \zeta)=\prod_{\sigma(t, \zeta) \leq t_{k}<t}\left(1+b_{k}\right)^{-1} Q(t, \zeta) .
\end{aligned}
$$

In fact, $v(t)$ is continuous on each interval $\left(t_{k}, t_{k+1}\right]$, and in view of $v\left(\bar{t}_{k}^{+}\right)=\left(1+b_{k}\right) v\left(\bar{t}_{k}\right)$, it follows that for all $t \geq t_{0}$,

$$
\begin{aligned}
& w\left(t_{k}^{+}\right)=\prod_{t_{0} \leq t_{j} \leq t_{k}}\left(1+b_{j}\right)^{-1} v\left(t_{k}^{+}\right)=\prod_{t_{0} \leq t_{j}<t_{k}}\left(1+b_{j}\right)^{-1} v\left(t_{k}\right)=w\left(t_{k}\right), \\
& w\left(t_{k}^{-}\right)=\prod_{t_{0} \leq t_{j} \leq t_{k-1}}\left(1+b_{j}\right)^{-1} v\left(t_{k}^{-}\right)=\prod_{t_{0} \leq t_{j}<t_{k}}\left(1+b_{j}\right)^{-1} v\left(t_{k}\right)=w\left(t_{k}\right)
\end{aligned}
$$

which implies that $w(t)$ is continuous on $\left[t_{0},+\infty\right)$.

$$
\begin{aligned}
& \frac{\mathrm{d}}{\mathrm{d} t}\left[w(t)+\int_{\alpha}^{\beta} G(t, \xi) w(\rho(t, \xi)) \mathrm{d} \eta(\xi)\right]+b_{0} a(t) w(t)+b_{0} \sum_{i=1}^{l} B_{i}(t) w\left(\tau_{i}(t)\right)+C \int_{\gamma}^{\delta} Q_{0}(t, \zeta) w(\sigma(t, \zeta)) \mathrm{d} \omega(\zeta) \\
& =\frac{\mathrm{d}}{\mathrm{d} t}\left[\prod_{t_{0} \leq t_{k}<t}\left(1+b_{k}\right)^{-1} v(t)+\int_{\alpha}^{\beta} \prod_{\rho(t, \xi) \leq t_{k}<t}\left(1+b_{k}\right)^{-1} g(t, \xi) \prod_{t_{0} \leq t_{k}<\rho(t, \xi)}\left(1+b_{k}\right)^{-1} v(\rho(t, \xi)) \mathrm{d} \eta(\xi)\right] \\
& \quad+b_{0} a(t) \prod_{t_{0} \leq t_{k}<t}\left(1+b_{k}\right)^{-1} v(t)+b_{0} \sum_{i=1}^{l} \prod_{\tau_{i}(t) \leq t_{k}<t}\left(1+b_{k}\right)^{-1} b_{i}(t) \prod_{t_{0} \leq t_{k}<\tau_{i}(t)}\left(1+b_{k}\right)^{-1} v\left(\tau_{i}(t)\right) \\
& \quad+C \int_{\gamma}^{\delta} \prod_{\sigma(t, \zeta) \leq t_{k}<t}\left(1+b_{k}\right)^{-1} Q(t, \zeta) \prod_{t_{0} \leq t_{k}<\sigma(t, \zeta)}\left(1+b_{k}\right)^{-1} v(\sigma(t, \zeta)) \mathrm{d} \omega(\zeta) \\
& =\prod_{t_{0} \leq t_{k}<t}\left(1+b_{k}\right)^{-1}\left(\frac{\mathrm{d}}{\mathrm{d} t}\left[v(t)+\int_{\alpha}^{\beta} g(t, \xi) v(\rho(t, \xi)) \mathrm{d} \eta(\xi)\right]+b_{0} a(t) v(t)\right. \\
& \left.\quad+b_{0} \sum_{i=1}^{l} b_{i}(t) v\left(\tau_{i}(t)\right)+C \int_{\gamma}^{\delta} Q(t, \zeta) v(\sigma(t, \zeta)) \mathrm{d} \omega(\zeta)\right) \leq 0,
\end{aligned}
$$

which implies that $w(t)$ is a positive solution. 
Now in inequality (7), set

$$
y(t)=w(t)+\int_{\alpha}^{\beta} G(t, \xi) w(\rho(t, \xi)) \mathrm{d} \eta(\xi) .
$$

Hence we have

$$
y^{\prime}(t)+b_{0} a(t) w(t)+b_{0} \sum_{i=1}^{l} B_{i}(t) w\left(\tau_{i}(t)\right)+C \int_{\gamma}^{\delta} Q_{0}(t, \zeta) w(\sigma(t, \zeta)) \mathrm{d} \omega(\zeta) \leq 0, \quad t \geq t_{0}, t \neq \bar{t}_{k} .
$$

For $t \geq t_{0}, t=\bar{t}_{k}, k=1,2, \cdots$, since $w(t)$ is continuous on $\left[t_{0},+\infty\right)$ and $G\left(\bar{t}_{k}^{+}, \xi\right)=G\left(\bar{t}_{k}, \xi\right)$, it is easy to verify that

$$
y\left(\bar{t}_{k}^{+}\right)=y\left(\bar{t}_{k}\right) .
$$

From inequality (9) and (10), it is easy to see that $y(t)$ is nonincreasing on $\left[t_{0},+\infty\right)$, so we have that $\lim _{t \rightarrow \infty} y(t)=L$.

Now we discuss $L$.

1) If we suppose that $L=-\infty$, then $\lim _{t \rightarrow \infty} y(t)=-\infty$. From inequality (8), we can get that $w(t)$ is unbounded; consequently, there exists $\left\{s_{k}: k \rightarrow \infty, s_{k} \rightarrow \infty\right\}$ such that $y\left(s_{k}\right)<0, w\left(s_{k}\right)=\max _{r \in\left[t_{0}, s_{k}\right]}\{w(r)\}$. Therefore $y\left(s_{k}\right)=w\left(s_{k}\right)+\int_{\alpha}^{\beta} G\left(s_{k}, \xi\right) w\left(\rho\left(s_{k}, \xi\right)\right) \mathrm{d} \eta(\xi) \geq\left[1+\int_{\alpha}^{\beta} G\left(s_{k}, \xi\right) \mathrm{d} \eta(\xi)\right] w\left(s_{k}\right) \geq 0$. This contradicts $y\left(s_{k}\right)<0$.

2) If we suppose that $L \neq 0$ is limited, then integrating inequality (9) from $t_{0}$ to $t$, Noting that $-\eta(\xi)$ is nondecreasing, then we can obtain

$$
\int_{t_{0}}^{t} b_{0} a(s) w(s) \mathrm{d} s+b_{0} \sum_{i=1}^{l} \int_{t_{0}}^{t} B_{i}(s) w\left(\tau_{i}(s)\right) \mathrm{d} s+C \int_{t_{0}}^{t} \mathrm{~d} s \int_{\gamma}^{\delta} Q_{0}(s, \zeta) w(\sigma(s, \zeta)) \mathrm{d} \omega(\zeta) \leq y\left(t_{0}\right)-y(t) .
$$

This implies that $w(t) \rightarrow 0$; hence, we have $y(t) \rightarrow 0$. This contradicts $L \neq 0$.

It follows that $L=0$. Since $y(t)$ is nonincreasing, then $y(t)>0$.

Noting that $-\eta(\xi)$ is nondecreasing, then we can obtain

$$
\begin{aligned}
& \begin{aligned}
w(t) & =y(t)-\int_{\alpha}^{\beta} G(t, \xi) w(\rho(t, \xi)) \mathrm{d} \eta(\xi) \\
& =y(t)-\int_{\alpha}^{\beta} G(t, \xi)\left[y(\rho(t, \xi))-\int_{\alpha}^{\beta} G(\rho(t, \xi), \xi) w(\rho(\rho(t, \xi), \xi)) \mathrm{d} \eta(\xi)\right] \mathrm{d} \eta(\xi) \\
& \geq y(t)-\int_{\alpha}^{\beta} G(t, \xi) y(\rho(t, \xi)) \mathrm{d} \eta(\xi) \geq\left(1-\int_{\alpha}^{\beta} G(t, \xi) \mathrm{d} \eta(\xi)\right) y(t) \geq\left(1-h_{0}\right) y(t),
\end{aligned} \\
& w(\sigma(t, \zeta)) \geq\left(1-h_{0}\right) y(\sigma(t, \zeta)) \geq\left(1-h_{0}\right) y(t-\imath) .
\end{aligned}
$$

From inequality (8), we get $y(t)<w(t)$. Then from (9), we obtain

$$
y^{\prime}(t)+b_{0} a(t) y(t)+C\left(1-h_{0}\right) y(t-\imath) \int_{\gamma}^{\delta} Q_{0}(t, \zeta) \mathrm{d} \omega(\zeta) \leq 0, t \geq t_{0}, t \neq \overline{t_{k}} .
$$

Hence, we can obtain that $y(t) \geq 0$ is an eventually positive solution of differential inequality (10), (11). But according to Lemma 2.2 (where $Q_{0}(t, \zeta)=\prod_{\sigma(t, \zeta) \leq t_{k}<t}\left(1+b_{k}\right)^{-1} Q(t, \zeta), d_{k}=0$ ) and condition (5), the differential inequality (10), (11) has no eventually positive solution. This is a contradiction. This ends the proof of the theorem.

\section{Oscillation Properties of the Problem (1), (4)}

The following theorem is the second main result of this article.

Theorem 3.1. Suppose that conditions (H1)-(H3) and the following conditions (12) hold

$$
\liminf _{t \rightarrow+\infty} \int_{t-l}^{t} \exp \left(\int_{s-l}^{s} b_{0} a(r) \mathrm{d} r\right) \prod_{\sigma(t, \zeta) \leq t_{k}<t}\left(1+b_{k}\right)^{-1} \mathrm{~d} s \int_{\gamma}^{\delta} C\left(1-h_{0}\right) Q(s, \zeta) \mathrm{d} \omega(\zeta)>\frac{1}{\mathrm{e}} .
$$


Then every solution of the problem (1), (4) oscillates in $G$.

Proof. Suppose that the assertion is not true and $u(t, x)$ is a non-oscillatory solution of problem (1), (4) in G. Without loss of generality, we may assume that there exists a $t_{0}>0$ such that $u(t, x)>0, u(\rho(t, \xi), x)>0$, $u\left(\tau_{i}(t), x\right)>0, i=1,2, \cdots, l, u(\sigma(t, \zeta), x)>0$, for any $(t, x) \in\left[t_{0},+\infty\right) \times \Omega$.

For $t \geq t_{0}, t \neq \bar{t}_{k}, k=1,2, \cdots$, multiplying (1) by $\varphi(x)$ and then integrating it with respect to $x$ over $\Omega$ yields

$$
\begin{aligned}
& \frac{\mathrm{d}}{\mathrm{d} t}\left[\int_{\Omega} \varphi(x) u(t, x) \mathrm{d} x+\int_{\alpha}^{\beta} g(t, \xi) \mathrm{d} \eta(\xi) \int_{\Omega} \varphi(x) u(\rho(t, \xi), x) \mathrm{d} x\right] \\
& =a(t) \int_{\Omega} \varphi(x) \Delta u \mathrm{~d} x+\sum_{i=1}^{l} b_{i}(t) \int_{\Omega} \varphi(x) \Delta u\left(\tau_{i}(t), x\right) \mathrm{d} x \\
& \quad-\int_{\gamma}^{\delta} \mathrm{d} \omega(\zeta) \int_{\Omega} \varphi(x) q(t, x, \zeta) f(u(\sigma(t, \zeta), x)) \mathrm{d} x+\int_{\Omega} \varphi(x) F(t, x) \mathrm{d} x .
\end{aligned}
$$

By Green's formula and the boundary condition, we have

$$
\int_{\Omega} \varphi(x) \Delta u \mathrm{~d} x-\int_{\Omega} u \Delta \varphi(x) \mathrm{d} x=\int_{\partial \Omega} \frac{\partial \varphi}{\partial n} u \mathrm{~d} s-\int_{\partial \Omega} \frac{\partial u}{\partial n} \varphi \mathrm{d} s=-\int_{\partial \Omega} c \varphi \cdot u \mathrm{~d} s+\int_{\partial \Omega} c u \cdot \varphi \mathrm{d} s=0 .
$$

It follows that

$$
\begin{aligned}
& \int_{\Omega} \varphi(x) \Delta u \mathrm{~d} x=\int_{\Omega} u \Delta \varphi(x) \mathrm{d} x=-b_{0} \int_{\Omega} u \varphi(x) \mathrm{d} x, \\
& \int_{\Omega} \varphi(x) \Delta u\left(\tau_{i}(t), x\right) \mathrm{d} x=\int_{\Omega} u\left(\tau_{i}(t), x\right) \Delta \varphi(x) \mathrm{d} x=-b_{0} \int_{\Omega} u\left(\tau_{i}(t), x\right) \varphi(x) \mathrm{d} x .
\end{aligned}
$$

The rest of the proof is similar to the one in Theorem 2.1, so we omit it.

\section{Remarks and Examples}

Remarks. From the theoretical viewpoint, the results of this paper, uncovered the essential difference between partial differential equations with impulses, functional arguments and partial differential equations without impulses, functional arguments; from a practical standpoint, they are very convenient because these criteria only depend on the coefficients of the equations, impulsive term and the time-delays. The results of this article improve the results in the papers [17]-[19]. For example, paper [19] discussed the case with distributed deviating arguments; however, we consider a more complex case with continuous distributed deviating arguments.

The following are examples to illustrate the applicability of the conditions.

Example 4.1. Consider the equation

$$
\begin{aligned}
& \frac{\partial}{\partial t}\left[u(t, x)+\int_{0}^{\pi} \frac{\mathrm{e}^{-t-\xi}}{4} u\left(t+\xi-\frac{3 \pi}{2}, x\right) \mathrm{d}(-\xi)\right] \\
& =u^{2} \Delta u+\mathrm{e}^{t} u^{2}\left(t-\frac{\pi}{2}, x\right) \Delta u\left(t-\frac{\pi}{2}, x\right)-\int_{\frac{\pi}{2}}^{\frac{3 \pi}{2}}\left(x^{2}+1\right) \mathrm{e}^{t-\zeta} u(t-\zeta, x) \mathrm{e}^{u^{2}(t-\zeta, x)} \mathrm{d} \zeta \\
& +\cos x \sin t, \quad t>1, t \neq 2^{k},(t, x) \in \mathbb{R}^{+} \times(0, \pi)=G, \\
& u\left(\left(2^{k}\right)^{+}, x\right)-u\left(\left(2^{k}\right)^{-}, x\right)=\frac{1}{2^{k}} u\left(2^{k}, x\right), \quad k=1,2, \cdots,
\end{aligned}
$$

and the boundary condition

$$
u(0, t)=u(\pi, t)=0, t \in \mathbb{R}^{+} .
$$

Here $\quad N=1, \quad \Omega=(0, \pi), \quad g(t, \xi)=\frac{\mathrm{e}^{-t-\xi}}{4}, \quad \rho(t, \xi)=t+\xi-\frac{3 \pi}{2}, \quad \eta(\xi)=-\xi, \quad a(t)=1, \quad b_{1}(t)=\mathrm{e}^{t}$, $h_{1}(u)=u^{2}, t_{k}=2^{k}, \tau_{1}(t)=t-\frac{\pi}{2}, q(t, x, \zeta)=\left(x^{2}+1\right) \mathrm{e}^{t-\zeta}, \quad f(u)=u \mathrm{e}^{u^{2}}, \sigma(t, \zeta)=t-\zeta$,

$F(t, x)=\cos x \sin t, \quad c=1$. It is easy to verify that the conditions (H1)-(H3) and the condition of Theorem 2.1 are satisfied. Hence all solutions of the above problem oscillate. 
Example 4.2. Consider the equation

$$
\begin{aligned}
& \frac{\partial}{\partial t}\left[u(t, x)+\int_{\frac{3 \pi}{2}}^{\frac{5 \pi}{2}} \frac{\mathrm{e}^{-t-\xi}}{4} u(t-\xi, x) \mathrm{d}(-\xi)\right] \\
& =u^{2} \Delta u+\mathrm{e}^{t} u^{2}\left(t-\frac{\pi}{2}, x\right) \Delta u\left(t-\frac{\pi}{2}, x\right)-\int_{\pi}^{2 \pi}\left(x^{2}+1\right) \mathrm{e}^{t-\zeta} u(t-\zeta, x) \mathrm{e}^{u^{2}(t-\zeta, x)} \mathrm{d} \zeta \\
& \quad+\cos x \sin t, \quad t>1, t \neq 2^{k},(t, x) \in \mathbb{R}^{+} \times(0, \pi)=G, \\
& \quad u\left(\left(2^{k}\right)^{+}, x\right)-u\left(\left(2^{k}\right)^{-}, x\right)=\frac{1}{2^{k}} u\left(2^{k}, x\right), \quad k=1,2, \cdots,
\end{aligned}
$$

with the boundary condition

$$
\frac{\partial u}{\partial n}(0, t)+u(0, t)=0, \quad \frac{\partial u}{\partial n}(\pi, t)+u(\pi, t)=0, t \in \mathbb{R}^{+} .
$$

Here $N=1, \Omega=(0, \pi), \quad g(t, \xi)=\frac{\mathrm{e}^{-t-\xi}}{4}, \rho(t, \xi)=t-\xi, \eta(\xi)=-\xi, \quad a(t)=1, \quad b_{1}(t)=\mathrm{e}^{t}, \quad h_{1}(u)=u^{2}$, $t_{k}=2^{k}, \tau_{1}(t)=t-\frac{\pi}{2}, q(t, x, \zeta)=\left(x^{2}+1\right) \mathrm{e}^{t-\zeta}, f(u)=u \mathrm{e}^{u^{2}}, \sigma(t, \zeta)=t-\zeta, \quad F(t, x)=\cos x \sin t, \quad c=1$. It is easy to verify that the conditions (H1)-(H3) and the condition of Theorem 3.1 are satisfied. Hence all solutions of the above problem oscillate.

\section{References}

[1] Yoshida, N. (1986) Oscillation of Nonlinear Parabolic Equations with Functional Arguments. Hiroshima Mathematical Journal, 16, 305-314.

[2] Minchev, P.E. and Bainov, D.D. (1988) Oscillation of the Solutions of Parabolic Differential Equations of Neutral Type. Applied Mathematics and Computation, 28, 97-111. http://dx.doi.org/10.1016/0096-3003(88)90089-6

[3] Liu, A.P., Yu, W.H. and Ding, Y.H. (2001) Necessary and Sufficient Conditions for Oscillations of Nonlinear Parabolic Partial Differential Equations. Pure and Applied Mathematics, 18, 86-89.

[4] Yang, Q.G. (2004) Oscillations of Solutions of a Class of Nonlinear Neutral Partial Differential Equations. Indian Journal of Pure \& Applied Mathematics, 35, 3-22.

[5] Shoukaku, Y. and Yoshida, N. (2010) Oscillations of Nonlinear Hyperbolic Equations with Functional Arguments via Riccati Method. Applied Mathematics and Computation, 217, 143-151. http://dx.doi.org/10.1016/j.amc.2010.05.030

[6] Lakshmikantham, V., Bainov, D.D. and Simeonov, P.S. (1989) Impulsive Differential Inequalities. In: Lakshmikantham, V., Bainov, D.D. and Simeonov, P.S., Eds., Theory of Impulsive Differential Equations, World Scientific Publishing Co. Pte. Ltd., Singapore City, 32-35.

[7] Erbe, L., Freedman, H., Liu, X.Z. and Wu, J.H. (1991) Comparison Principles for Impulsive Parabolic Equations with Application to Models of Single Species Growth. Journal of the Australian Mathematical Society, 32, 382-400. http://dx.doi.org/10.1017/S033427000000850X

[8] Bainov, D.D. and Minchev, P.E. (1998) Forced Oscillations of Solutions of Impulsive Nonlinear Parabolic Differential-Difference Equations. Journal of the Korean Mathematical Society, 35, 881-890.

[9] Bainov, D.D. and Minchev, P.E. (1996) Oscillation of Solutions of Impulsive Nonlinear Parabolic Differential-Difference Equations. International Journal of Theoretical Physics, 35, 207-215. http://dx.doi.org/10.1007/BF02082944

[10] Fu, X.L., Liu, X.Z. and Sivaloganathan, S. (2002) Oscillation Criteria for Impulsive Parabolic Differential Equations with Delay. Journal of Mathematical Analysis and Applications, 268, 647-664. http://dx.doi.org/10.1006/jmaa.2001.7840

[11] Liu, A.P., Xiao, L. and He, M.X. (2004) Oscillation of Nonlinear Hyperbolic Differential Equations with Impulses. Nonlinear Oscillations, 7, 425-431. http://dx.doi.org/10.1007/s11072-005-0022-X

[12] Liu, A.P., Ma, Q.X. and He, M.X. (2006) Oscillation of Nonlinear Impulsive Parabolic Equations of Neutral Type. Rocky Mountain Journal of Mathematics, 36, 1011-1026. http://dx.doi.org/10.1216/rmjm/1181069442

[13] Tanaka, S. and Yoshida, N. (2005) Forced Oscillation of Certain Hyperbolic Equations with Continuous Distributed Deviating Arguments. Annales Polonici Mathematici, 85, 37-54. http://dx.doi.org/10.4064/ap85-1-4 
[14] Luo, L.P., Gao, Z.H. and Ouyang, Z.G. (2006) Oscillation of Nonlinear Neutral Parabolic Partial Functional Differential Equations with Continuous Distribution Delay. Mathematica Applicata, 19, 651-655.

[15] Shoukaku, Y. (2011) Forced Oscillatory Result of Hyperbolic Equations with Continuous Distributed Deviating Arguments. Applied Mathematics Letters, 24, 407-411. http://dx.doi.org/10.1016/j.aml.2010.10.012

[16] Yan, J.R. and Kou, C.H. (2001) Oscillation of Solutions of Impulsive Delay Differential Equations. Journal of Mathematical Analysis and Applications, 254, 358-370. http://dx.doi.org/10.1006/jmaa.2000.7112

[17] Deng, L.H. and Ge, W.G. (2001) Oscillation of the Solutions of Parabolic Equations with Impulses. Acta Mathematica Sinica, 44, 501-506.

[18] Yan, J.R. (2004) Oscillation Properties of Solutions for Impulsive Delay Parabolic Equations. Acta Mathematica Sinica, 47, 579-586.

[19] Liu, A.P., Liu, T. and Zou, M. (2011) Oscillation of Nonlinear Impulsive Parabolic Differential Equations of Neutral Type. Rocky Mountain Journal of Mathematics, 41, 833-850. http://dx.doi.org/10.1216/RMJ-2011-41-3-833 